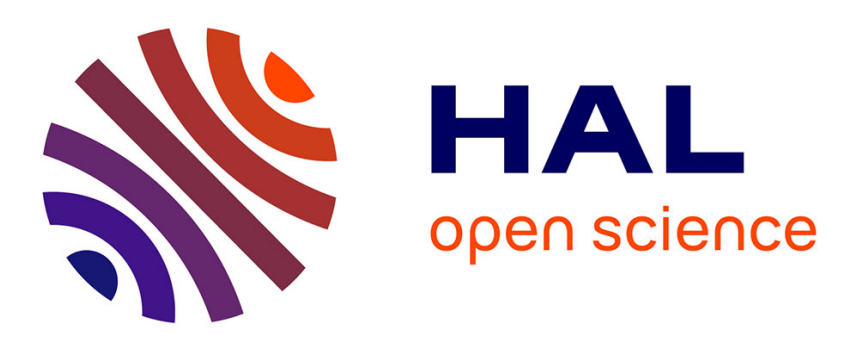

\title{
Etude des possibilités de détection précoce du viroïde des tubercules fusiformes de la pomme de terre
}

\author{
Guy Macquaire, Marie Monsion, Jean Dunez
}

\section{To cite this version:}

Guy Macquaire, Marie Monsion, Jean Dunez. Etude des possibilités de détection précoce du viroïde des tubercules fusiformes de la pomme de terre. Agronomie, 1987, 7 (8), pp.639-645. hal-00885037

\section{HAL Id: hal-00885037 \\ https://hal.science/hal-00885037}

Submitted on 1 Jan 1987

HAL is a multi-disciplinary open access archive for the deposit and dissemination of scientific research documents, whether they are published or not. The documents may come from teaching and research institutions in France or abroad, or from public or private research centers.
L'archive ouverte pluridisciplinaire HAL, est destinée au dépôt et à la diffusion de documents scientifiques de niveau recherche, publiés ou non, émanant des établissements d'enseignement et de recherche français ou étrangers, des laboratoires publics ou privés. 


\title{
Etude des possibilités de détection précoce du viroïde des tubercules fusiformes de la pomme de terre
}

GuY MACQUAIRE, Marie MONSION \& Jean DUNEZ

I.N.R.A., Station de Pathologie végétale, Centre de Recherches de Bordeaux, B.P. 131, F 33140 Pont-de-la-Maye

Les effets du PSTV ont été étudiés sur plusieurs cultivars de pomme de terre infectés artificiellement et maintenus en serre isolée. Dans ces conditions de culture, l'infection a peu d'effet sur les organes aériens ; en revanche, on observe un effet très marqué sur la taille et les facultés germinatives des tubercules de certaines variétés, notamment " Belle de Fontenay ".

L'évolution de l'infection dans la plante a été suivie tout au long du cycle végétatif, dans les feuilles, puis dans les tubercules. Le PSTV a été recherché par la technique d'hybridation moléculaire utilisant une sonde constituée d'un plasmide contenant une copie complète de PSTV et marquée au ${ }^{32} \mathrm{P}$ par « nick translation ». La présence du PSTV peut être mise en évidence très précocement après l'inoculation et il existe une parfaite corrélation entre les résultats des tests effectués sur les parties aériennes et sur les tubercules. Cependant, une certaine hétérogénéité de la distribution du PSTV pose le problème de l'échantillonnage.

L'intérêt et la sensibilité de la technique d'hybridation moléculaire (qui permet de détecter régulièrement 15 à $30 \mathrm{pg}$ de viroïde) ont été confirmés. Cette méthode apparaît particulièrement adaptée à une détection précoce du viroïde aussi bien dans les organes aériens que dans les tubercules.

Mots clés additionnels : Hybridation moléculaire, sonde, cDNA, marquage radioactif.

\begin{abstract}
Possibilities of early detection of potato spindle tuber viroid (PSTV).
Several potato cultivars were artificially inoculated. Under our culture conditions in an isolated greenhouse, PSTV appeared to have little effect on the above-ground part of the plant ; by contrast, it has marked effect on tuber production and quality : the size and germination of tubers of some cultivars were particularly affected (Belle de Fontenay). Spread of PSTV in the plant from the inoculation point was followed by sampling of leaves during the vegetation period, and subsequently of tubers. PSTV was detected by a molecular hybridization technique using a ${ }^{32} \mathrm{P}$-labelled probe consisting of a plasmid containing a full-length copy of PSTV. Presence of PSTV was detected as early as 9 days after inoculation and the results point to a good correlation between the results of the tests carried out on leaves and on tubers. Nevertheless uneven distribution of the viroid in leaves and tubers was observed, which poses the problem of sampling. The reliability and sensitivity of the molecular hybridization technique (which detects as low as $15-30 \mathrm{pg}$ viroid) were confirmed : this technique appears to be perfectly adapted to early detection of PSTV in leaves and tubers of infected potato plants.
\end{abstract}

Additional key words : Molecular hybridization, $c D N A$, probe, radioactive labelling.

\section{INTRODUCTION}

Le viroïde des tubercules fusiformes de la pomme de terre (Potato spindle tuber viroid : PSTV) est connu pour induire de sévères symptômes dans différentes variétés sous certaines conditions de culture et de climat.

C'est essentiellement pour l'Europe un agent de quarantaine ; les pays dans lesquels il n'a pas encore été introduit développent des mesures très strictes pour éviter son introduction. Il est donc important de dispo- ser de techniques de détection présentant un haut niveau de sensibilité mais également capables d'assurer une détection aussi précoce que possible de l'agent pathogène. Différentes méthodes ont été successivement appliquées à la détection du PSTV. Aux techniques biologiques fondées sur les propriétés infectieuses du PSTV et la possibilité de le transmettre mécaniquement sur des cultivars sensibles de tomate ont succédé une technique électrophorétique (MORRIS \& SMITH, 1976), puis une technique d'hybridation moléculaire (OWENS \& DIENER, 1981). Récemment, il a été proposé 
d'utiliser une technique d'électrophorèse bidirectionnelle, permettant de mieux séparer le viroïde des acides nucléiques cellulaires, assortie d'une coloration au nitrate d'argent apportant une sensibilité accrue (SCHUMACHER et al., 1985 ; HUTTINGA, comm. pers.).

En ce qui nous concerne, nous avons choisi d'utiliser la technique d'hybridation moléculaire, plus sensible et plus facile à mettre en œuvre que l'électrophorèse. Cette technique a, en outre, comme l'avaient souligné OWENS \& DIENER (1981), l'avantage de pouvoir être utilisée avec les feuilles et les tubercules. Nous avons recherché dans quelles conditions une détection précoce et fiable du viroïde était possible dans les organes aériens de la plante dans les semaines suivant l'infection ou dans les tubercules récoltés sur des plants inoculés en début de végétation.

Ce travail nous a également permis d'observer l'effet du PSTV sur plusieurs variétés françaises de pomme de terre lors d'un essai réalisé sous conditions totales d'isolement, en serre étanche. Les résultats de ce travail ont d'abord montré que l'infection par le PSTV, bien que passant à peu près inaperçue sur les organes aériens de la plante l'année de l'infection, a un effet très marqué sur la récolte et notamment la qualité des tubercules. L'infection est décelable très peu de temps après l'inoculation mais la généralisation de l'infection aux organes aériens et à l'ensemble des tubercules n'est pas totale : ceci pose le problème d'une définition précise de l'échantillonnage.

\section{MATÉRIEL ET MÉTHODES}

\section{A. Matériel}

\section{Cultivars de pomme de terre}

Au printemps 1984, sept variétés : « Bintje », « Saskia », " Rosewald », « Bea », « Belle de Fontenay» et «BF 15 », furent inoculées pour vérifier leur sensibilité au PSTV. "Belle de Fontenay » et «BF 15 » (issue du croisement de Belle de Fontenay par Flavia) sont apparues particulièrement sensibles et la variété BF 15 plus largement cultivée a été finalement retenue pour la réalisation de ce travail.

Les résultats de quelques essais réalisés avec « Belle de Fontenay » et « Bintje » seront également mentionnés.

\section{Souches du PSTV}

La souche du PSTV utilisée, décrite par MACQUAIRE et al. (1984), est propagée sur des tomates cv. Rutgers, cultivées en chambre climatique isolée, maintenue à une température (jour/nuit) de $28 / 25^{\circ} \mathrm{C}$. Cette souche peut être considérée comme de sévérité moyenne compte tenu des symptômes induits sur tomate.

\section{B. Méthodes}

\section{Culture des pommes de terre. Inoculation par le PSTV. Prélèvement des échantillons}

Cinquante tubercules de "BF 15 », classe super ELITE, ont été mis en culture dans des banquettes placées dans une petite serre isolée étanche.

Vingt jours après plantation, chaque pousse possède
2-3 feuilles ; 24 plantes ont alors été inoculées par insertion (dans 2 tiges par plante) de 2 fragments d'écorce de tomates infectées par le PSTV. Les plantes restantes ont reçu, dans les mêmes conditions, des implants de tomates saines.

Le premier prélèvement de feuilles a lieu le jour de l'inoculation ; les suivants $9,17,28,63$ et 80 jours après inoculation (tabl. 2). Les prélèvements de feuilles sont réalisés à la fois sur les tiges inoculées et non inoculées.

Deux jours après le dernier prélèvement, les tubercules sont récoltés et les fannes incinérées.

Outre les prélèvements foliaires, les tubercules ont été analysés à partir d'échantillons comportant soit la chair, soit un œil accompagné d'un peu de peau et de chair. Après germination, les prélèvements ont porté sur les très jeunes feuilles portées par les nouvelles pousses.

\section{Analyse des échantillons par hybridation moléculaire}

Les échantillons sont broyés, à l'aide d'un broyeur à rouleaux, dans un tampon glycine $0,2 \mathrm{M}$, phosphate disodique $0,1 \mathrm{M}, \mathrm{NaCl} 0,6 \mathrm{M}$, SDS 1 p. 100, additionné de phénol et de chloroforme. L'extrait est centrifugé à $15000 \mathrm{t} / \mathrm{mn}$ pendant $10 \mathrm{mn}: 5 \mu \mathrm{l}$ de surnageant sont dénaturés avec $50 \mathrm{mM}$ d'hydroxy-méthyl mercure (BAILEY \& DAVIDSON, 1976) et déposés sur une membrane de nitrocellulose dans un « hybridot " BRL. Les membranes séchées sont cuites $2 \mathrm{~h}$ à $80^{\circ} \mathrm{C}$ sous vide, puis enfermées dans un sac de polyéthylène scellé. Les membranes ainsi préparées peuvent être conservées plusieurs mois à $4{ }^{\circ} \mathrm{C}$ avant hybridation. L'hybridation est réalisée avec une sonde constituée d'un plasmide pAV 401 contenant une copie complète du PSTV et marqué par nick translation avec l' $\alpha^{32} \mathrm{P}$ dCTP (Amersham). Les résultats sont observés par autoradiographie sur film Kodak X-OMAT AR après $24 \mathrm{~h}$ d'exposition. Les modalités détaillées de cette opération ont été décrites par MACQUAIRE et al. (1984).

\section{RÉSULTATS}

\section{A. Effet de l'infection sur la qualité de la récolte}

Aucun effet significatif n'a été observé sur les organes aériens l'année de l'inoculation. Par contre, comme cela a déjà été décrit (PFANNENSTIEL \& SLACK, 1980 ; KOWALSKA et al., 1980), les tubercules sont fortement affectés. Dans la majorité des cas, l'infection se traduit par une diminution du nombre de tubercules et une réduction de la taille (fig. 1: A à D). En plus des altérations déjà rapportées pour les autres variétés, tubercules en fuseau de petite taille aux yeux proéminents, la variété "Belle de Fontenay " présente une réaction particulière. En effet, dans certains cas, les tubercules peuvent présenter un profond dérèglement végétatif : ils ne connaissent pas de dormance et les yeux évoluent immédiatement en nouveaux tubercules dont les yeux se développent à leur tour en nouveaux petits tubercules. On peut ainsi observer plusieurs " générations " de tubercules sans développement d'organes aériens. Cette variété présente parfois des dérèglements végétatifs de cette nature en l'absence 

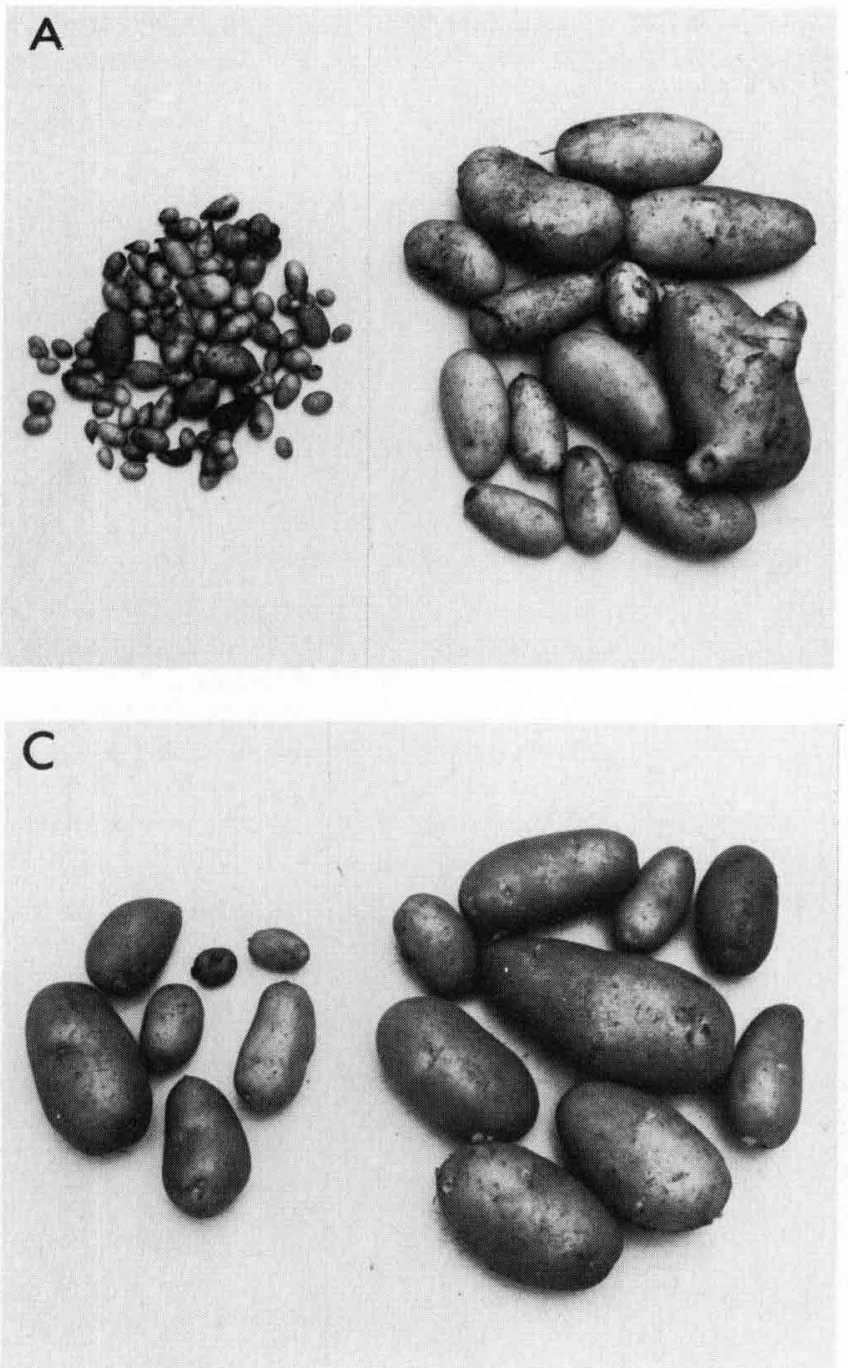

Figure 1

Symptômes morphologiques observés sur les tubercules de différentes variétés de pomme de terre infectées par le PSTV (à gauche). A droite, plantes saines.

A. Belle de Fontenay - B. Saskia - C. Roseval - D. Bintje.

d'infection par le PSTV : on doit donc être prudent dans l'attribution au PSTV de ces symptômes qui, dans notre essai, ne se sont jamais manifesté sur les pieds sains (fig. 2).

Une étude plus précise de la récolte a été réalisée sur BF 15 en 1985 (tabl. 1). Il n'a pas été possible d'observer d'effet réellement significatif au niveau du poids de la récolte, celui-ci présentant une grande variabilité d'un pied à l'autre. De plus, l'écart type, calculé sur 25 pieds témoins, est élevé $(25,7)$ et rend donc difficile une interprétation précise. Il paraît vraisemblable que l'infection par le PSTV n'a pas en première année d'effet clairement dépressif sur le poids de la récolte. Dans un certain nombre de cas, le nombre de tubercules est considérablement augmenté, leur taille étant réduite dans des proportions importantes. La récolte devient, le plus souvent, totalement invendable (fig. 1). Enfin, après conservation et levée de dormance, les propriétés germinatives des plants infectés sont très réduites. Cet effet est difficile à quantifier car, dans la majorité des cas, les tubercules de très petite taille ne germent pas du tout et le nombre de pousses développées à partir des tubercules de taille normale (issus de
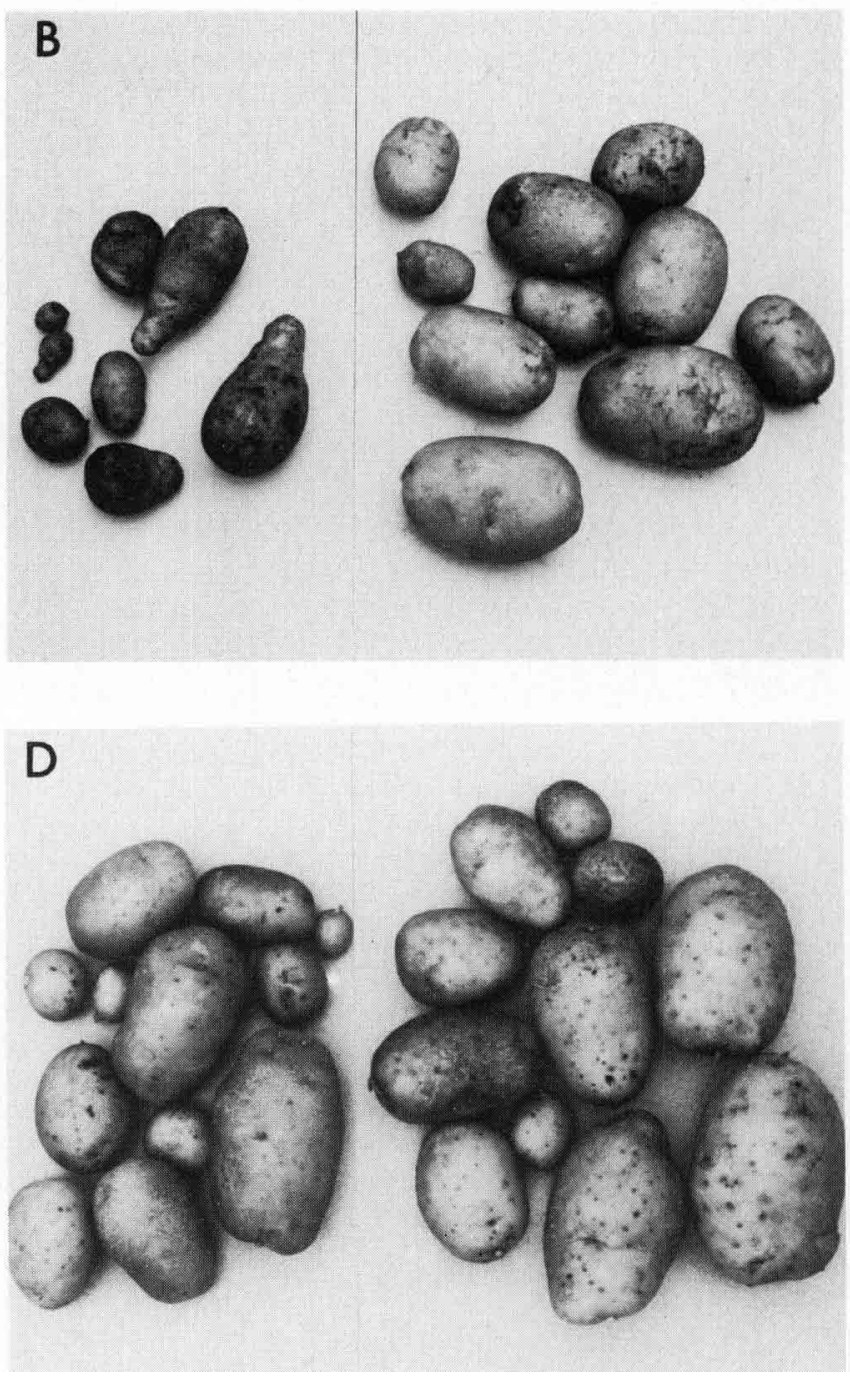

Morphological symptoms induced by PSTV on tubers of several potato cultivars on the left. On the right, healthy plants.

A. Belle de Fontenay - B. Saskia-C. Roseval-D. Bintje.

pieds infectés) est, en apparence, plus faible que la normale (fig. 3 A et B).

Neuf pieds inoculés sur 24 ont fourni une récolte en apparence non affectée ni dans le poids, ni dans la forme, le nombre ou la taille des tubercules (tabl. 1). Le contrôle de ces tubercules par hybridation moléculaire montrera que 7 d'entre eux étaient infectés : l'observation morphologique des tubercules ne peut donc être un critère d'infection même dans une variété sensible.

\section{B. Recherche du PSTV par hybridation moléculaire}

Les résultats de l'analyse des échantillons, récoltés comme nous l'avons indiqué au chapitre "Matériel et Méthodes ", sont portés sur le tabl. 2. Il est intéressant de noter que, dès le $9^{e}$ jour après l'inoculation, le PSTV a pu être décelé, chez 5 plantes, par analyse des feuilles développées sur les tiges inoculées. A 17 jours, les résultats n'ont pas évolué ; certains cas d'infection décelés 8 jours avant ne le sont plus : ceci s'explique par le fait que les échantillons ont pu être prélevés sur des tiges différentes. 


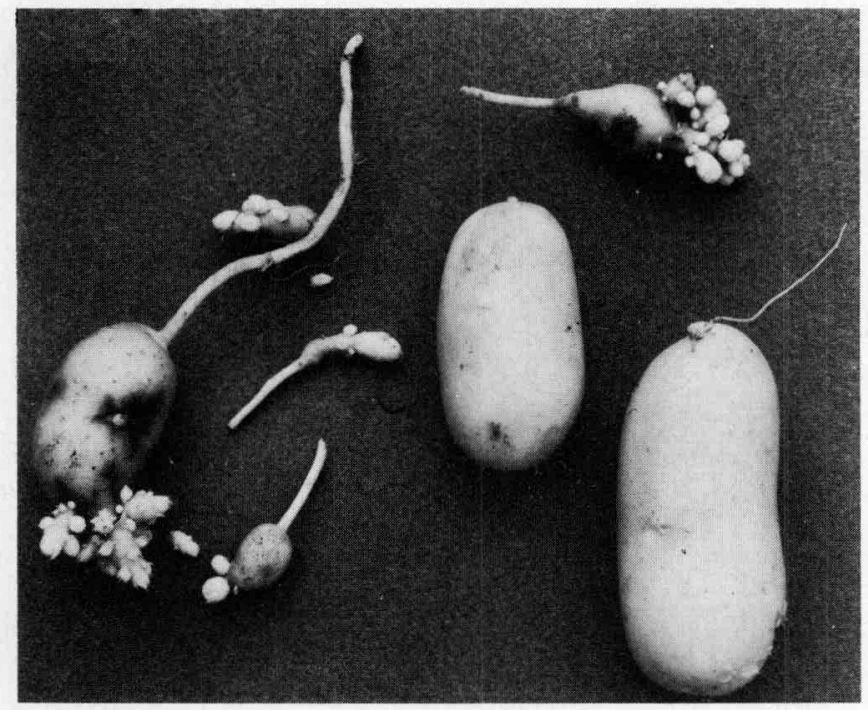

TABLEAU 1

Effet de l'infection par le PSTV sur la production de pommes de terre, variété $B F 15$.

Effect of PSTV infection on the crop of potato plants var BF 15 .

\begin{tabular}{cccc} 
Plant & $\begin{array}{c}\text { Symptoms } \\
\text { on tubers }\end{array}$ & $\begin{array}{c}\text { Number of } \\
\text { tubers }\end{array}$ & $\begin{array}{c}\text { Total weight } \\
\text { of tubers }\end{array}$ \\
\hline \hline $\begin{array}{c}\text { Numéro } \\
\text { du pied }\end{array}$ & $\begin{array}{c}\text { Aspect des } \\
\text { tubercules** }\end{array}$ & $\begin{array}{c}\text { Nb total de } \\
\text { tubercules }\end{array}$ & $\begin{array}{c}\text { Poids de } \\
\text { la récolte }\end{array}$ \\
\hline $\begin{array}{c}\text { Témoin* } \\
1\end{array}$ & - & 13 & 165,4 \\
2 & + & 9 & 180,5 \\
3 & - & 9 & 129,2 \\
4 & - & 10 & 165 \\
5 & - & 10 & 188 \\
6 & - & 10 & 142,4 \\
7 & - & 11 & 129,6 \\
8 & + & 11 & 125,1 \\
9 & - & 15 & 145,2 \\
10 & + & 16 & 127,5 \\
11 & +++ & 21 & 190,7 \\
12 & ++ & 26 & 147,2 \\
13 & +++ & 22 & 206,1 \\
14 & +++ & 16 & 218,8 \\
15 & +++ & 18 & 193,1 \\
16 & - & 35 & 141,1 \\
17 & ++ & 15 & 183,1 \\
18 & +++ & 22 & 140,7 \\
19 & +++ & 16 & 181,1 \\
20 & ++ & 28 & 272,4 \\
21 & +++ & 18 & 137 \\
22 & +++ & 28 & 169 \\
23 & +++ & 23 & 216 \\
24 & - & 40 & 174,4 \\
\hline \hline
\end{tabular}

* Moyenne de 25 pieds (mean of 25 plants).

** Etabli sur l'ensemble des tubercules (global notation of all tubers).

- $\quad$ aucun tubercule anormal (no abnormal tubers)

$+\quad$ quelques tubercules anormaux (a few abnormal tubers),

+ moins de 50 p. 100 de tubercules anormaux (less than $50 \%$ abnormal tubers),

+++ plus de 50 p. 100 de tubercules anormaux (more than $50 \%$ abnormal tubers).

Deux mois après l'inoculation, le PSTV a été décelé, si l'on considère l'ensemble des échantillons analysés, sur toutes les plantes sauf 3 . Il faut néanmoins noter, que le PSTV est parfois impossible à identifier, même sur les tiges inoculées. Ce résultat souligne le problème
Figure 2

Dérèglement végétatif observé sur tubercules de la variété Belle de Fontenay infectés par le PSTV.

Comparaison avec 2 tubercules sains (en bas à droite).

Disorders induced on Belle de Fontenay tubers by PSTV $(2$ healthy tubers on the right).

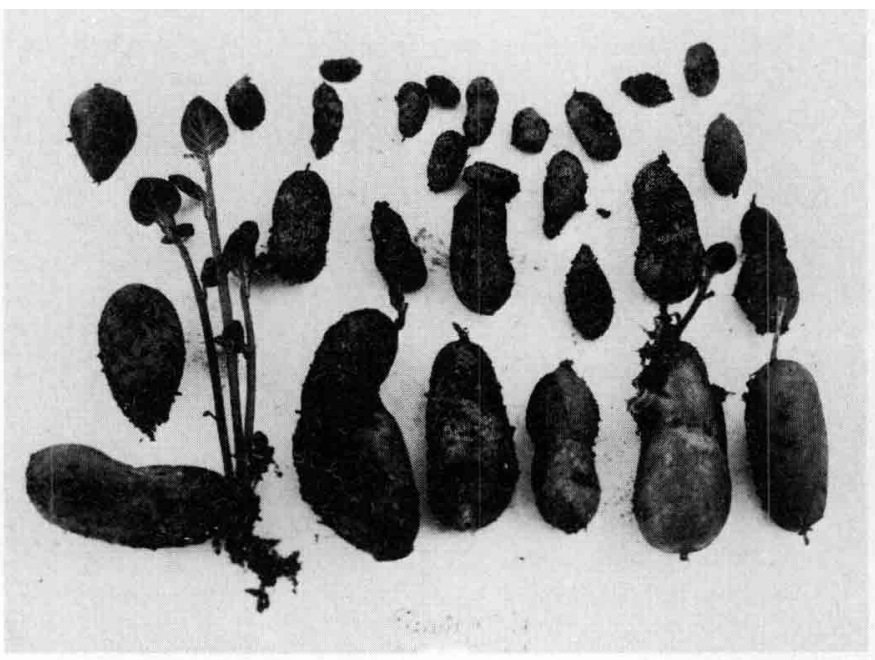

a)

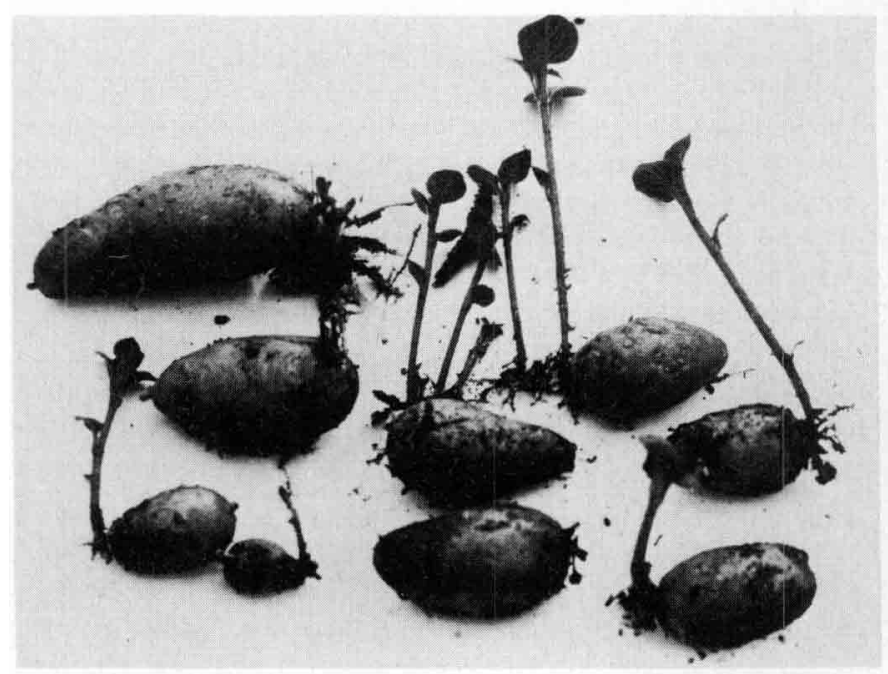

b)

Figure 3

Germination de tubercules de pomme de terre var BF 15 respectivement issus d'une plante infectée par le PSTV (3A) et saine ( $3 B$ ).

Germination of potato tubers var BF 15 from a PSTV infected plant (3A) and a healthy one (3B). 
TABLEAU 2

Détection du PSTV dans les plantes inoculées par hybridation moléculaire. PSTV detection by molecular hybridization in inoculated plants.

\begin{tabular}{|c|c|c|c|c|c|c|c|c|c|c|c|c|c|}
\hline \multirow{4}{*}{$\begin{array}{c}\mathrm{N}^{\circ} \\
\mathrm{du} \\
\text { pied }\end{array}$} & \multicolumn{13}{|c|}{ Nature de l'échantillon (type of sample) } \\
\hline & \multicolumn{11}{|c|}{ Feuilles (date de prélèvement en jours après inoculation) - Leaves (days after inoculation) } & \multicolumn{2}{|c|}{ Tubercules (tubers) } \\
\hline & \multirow[t]{2}{*}{0} & \multicolumn{2}{|c|}{9} & \multicolumn{2}{|c|}{17} & \multicolumn{2}{|c|}{28} & \multicolumn{2}{|c|}{63} & \multicolumn{2}{|c|}{80} & \multirow{2}{*}{$\mathrm{G}^{*}$} & \multirow{2}{*}{$\mathrm{C}^{*}$} \\
\hline & & I* & $\mathrm{NI}^{*}$ & I & NI & I & NI & I & NI & I & NI & & \\
\hline 1 & - & - & - & - & - & - & $-\cdots$ & + & - & +++ & ++ & + & - \\
\hline 2 & - & - & - & - & - & - & - & ++ & + & $++t$ & + & + & + \\
\hline 3 & - & + & - & - & - & ++ & - & + & - & 0 & 0 & + & + \\
\hline 4 & - & - & - & - & - & - & - & - & - & - & - & - & - \\
\hline 5 & - & - & - & - & - & - & - & ++ & ++ & ++ & $+t+$ & + & + \\
\hline 6 & - & - & - & - & - & - & - & - & - & 0 & - & + & + \\
\hline 7 & - & - & - & - & - & + & + & - & ++ & $t+$ & $+t$ & +++ & + \\
\hline 8 & - & - & - & - & - & - & - & - & + & ++ & $+t$ & $++t$ & + \\
\hline 9 & - & + & - & - & - & - & + & + & + & ++ & ++ & $t+t$ & + \\
\hline 12 & - & - & - & - & - & ++ & - & - & ++ & 0 & 0 & + & - \\
\hline 13 & - & - & - & - & - & - & - & - & ++ & $+t+$ & + & + & + \\
\hline 14 & - & + & + & - & - & - & - & +++ & ++ & $t+t$ & +++ & $++t$ & + \\
\hline 15 & - & + & - & + & - & - & + & + & +++ & +++ & $++t$ & $+t+$ & + \\
\hline 16 & - & - & - & - & - & - & - & - & - & 0 & - & + & + \\
\hline 17 & - & - & - & + & - & + & + & ++ & + & 0 & 0 & - & - \\
\hline 18 & - & - & - & - & - & - & + & - & +++ & + & ++ & ++ & + \\
\hline 19 & - & - & - & - & - & - & - & - & ++ & + & ++ & + & ++ \\
\hline 20 & - & - & - & + & - & + & - & + & + & 0 & 0 & + & + \\
\hline 21 & - & - & - & - & - & ++ & + & ++ & $++t$ & ++ & +++ & + & + \\
\hline 22 & - & - & - & - & - & - & + & + & + & +++ & 0 & +++ & + \\
\hline 23 & - & - & - & - & - & - & + & + & + & ++ & +++ & + & ++ \\
\hline 24 & - & - & - & - & - & + & - & - & + & + & + & + & + \\
\hline sain & - & - & - & - & - & - & - & - & - & - & - & - & - \\
\hline
\end{tabular}

*I : feuilles prélevées sur une tige inoculée par le PSTV (leaves harvested on an inoculated stem).

*NI : feuilles prélevées sur une tige non inoculée par le PSTV (leaves harvested on an uninoculated stem).

${ }^{*} \mathrm{G} \quad$ : prélèvement d'un germe accompagné d'épiderme (sample of one eye plus epidermis).

* $\mathrm{C} \quad$ : prélèvement de la chair (sample of tuber flesh).

$0 \quad$ : plante n'ayant pu être contrôlée (not tested).

: test négatif (no hybridization signal).

test faiblement positif (weakly positive hybridization signal)

$+\quad$ : test nettement positif (positive hybridization signal).

+++ : test fortement positif (strongly positive hybridization signal).

de l'échantillonnage dans une recherche précoce du viroïde.

Au dernier prélèvement, 80 jours après inoculation, tous les échantillons, prélevés ou non sur les tiges inoculées, sont clairement positifs sauf 3 plantes qui paraissent avoir échappé à l'infection.

Une analyse a été effectuée après récolte sur les tubercules, des prélèvements étant réalisés soit au niveau des germes et comprenant l'œil, la peau et un peu de chair du tubercule, soit loin des germes et ne comportant que la chair. Les 2 types d'echantillons ont donné, à 2 exceptions près, des résultats identiques, mais l'hybridation est plus forte lorsque les échantillons sont prélevés au niveau d'un œil, reflétant vraisemblablement une teneur plus élevée en viroïde. Les résultats et les niveaux d'hybridation sont alors tout à fait analogues à ceux observés avec les feuilles.

On peut alors se demander si tous les tubercules sont uniformément infectés. Pour tenter de répondre à cette question, 4 plantes ont été sélectionnées, 2 chez lesquelles le viroïde a été régulièrement mis en évidence ( $\mathrm{n}^{\circ} 15$ et 21 ), une chez laquelle le PSTV n'a été décelé que dans les tubercules et jamais durant la végétation $\left(n^{\circ} 16\right)$ et une enfin chez laquelle le PSTV n'a jamais été décelé $\left(n^{\circ} 4\right)$. Huit tubercules prélevés au hasard chez ces 4 plants ont été analysés individuellement. De plus, pour confirmer ces résultats, les tubercules, après analyse, ont été mis à germer et une nouvelle analyse a été réalisée sur les jeunes feuilles portées par les pousses. L'ensemble de ces résultats est porté sur la figure 4.

Il faut d'abord noter la parfaite corrélation entre les résultats obtenus sur les tubercules (T) et avec les pousses (F) qui en sont issues. Par ailleurs, les 8 tubercules des plants 15 et 21 chez lesquelles l'infection avait été déjà clairement mise en évidence sont tous infectés. Chez la plante $n^{\circ} 16,4$ tubercules sur 8 apparaissent infectés et chez la plante $n^{\circ} 4$ où aucune infection n'avait été décelée, 3 tubercules sur 8 sont nettement infectés. Ces résultats posent à nouveau le problème de la distribution inégale du PSTV et de l'échantillonnage.

\section{DISCUSSION - CONCLUSION}

A la fois pour une surveillance efficace au champ et pour un contrôle sûr et rapide des introductions, il est nécessaire de disposer de techniques sensibles permettant de déceler des infections récentes par le PSTV. 


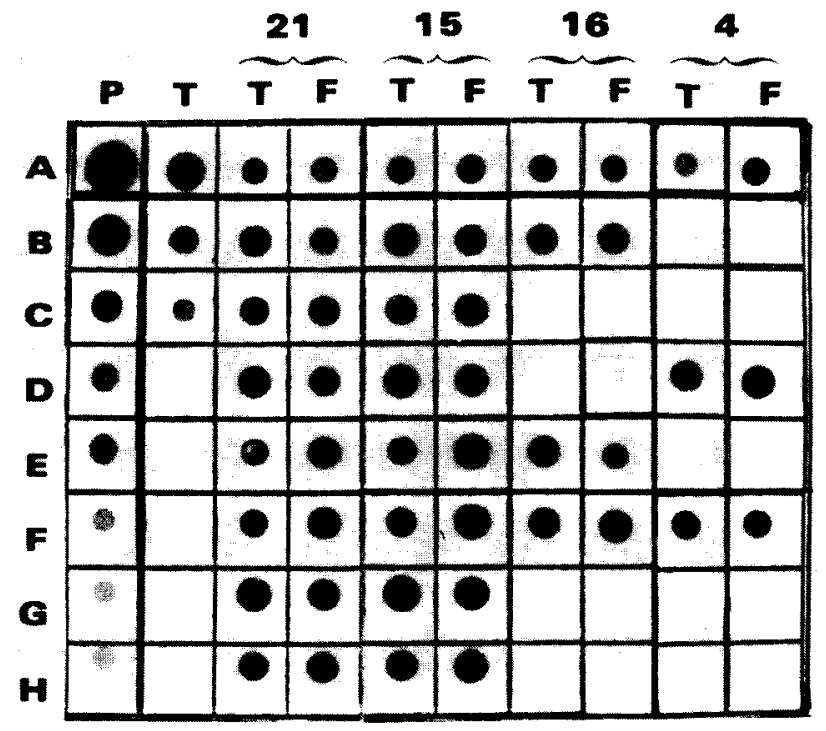

Figure 4

Détection du PSTV dans les tubercules $(T)$ et les pousses $(F)$ issues des tubercules produits par des plants inoculés.

Concentrations : $A$ à $G: 25-8,3-2,7-0,92-0,30-0,10-0,034 n g$.

$P$ : solution PSTV purifié

$T$ - $A$ à $D$ : dilution d'extrait de feuilles de pomme de terre infectées $E$ à $H$ : dilution d'extrait de feuilles de pomme de terre saines 21 : analyse de 8 tubercules $\left(A\right.$ à $H$ ) pied $n^{\circ} 21$

15 : analyse de 8 tubercules $(A$ à $H)$ pied $n^{\circ} 15$

16 : analyse de 8 tubercules $\left(A\right.$ à $H$ ) pied $n^{\circ} 16$

4 : analyse de 8 tubercules $\left(A\right.$ à $H$ ) pied $n^{\circ} 4$.

Detection of PSTV in tubers and shoots from tubers produced by $P S T V$ inoculated plants.

$P$ : purified PSTV

$T: A-D:$ dilution of infected leaf extracts

$E-H$ : dilution of healthy leaf extracts

21 : test of 8 tubers $(A-H)$ of plant $n^{\circ} 21$

15 : test of 8 tubers $(A-H)$ of plant $n^{\circ} 15$

16: test of 8 tubers $(A-H)$ of plant $n^{\circ} 16$

4 : test of 8 tubers $(A-H)$ of plant $n^{\circ} 4$

L'étude expérimentale réalisée démontre que le PSTV inoculé à un plant de pomme de terre l'envahit rapidement et que sa détection est possible quelques semaines, voire quelques jours après inoculation.

Cependant, le fait que, dans plusieurs cas, on ne retrouve plus le PSTV dans les feuilles des tiges inoculées alors qu'on le trouve dans les autres, illustre bien l'hétérogénéité de la distribution et la nécessité, pour une étude précise au champ, de travailler sur un échantillon composé de feuilles prélevées sur différentes tiges de la plante. Dans la très grande majorité des cas, l'infection est décelable moins de 2 mois après l'inoculation sur les organes aériens et se retrouve dans les tubercules. L'analyse des tubercules a clairement démontré que le PSTV pouvait être détecté aussi bien sur des échantillons constitués de la chair que sur ceux qui consistaient en un œil accompagné d'un peu d'épiderme. Cependant, dans la majorité des cas, les résultats sont plus nets avec ce dernier type d'échantillon soit (vraisemblablement) que la concentration en PSTV soit plus élevée, soit qu'il existe dans la chair du tubercule des principes inhibiteurs en plus grande quantité que dans les germes. Une fois de plus le problème de l'échantillonnage se pose. Lorsque l'infection s'est parfaitement établie dans la plante et que le PSTV peut être détecté régulièrement sur les organes aériens, tous les tubercules sont uniformément infectés ; en revanche, lorsque l'infection s'est plus mal établie et que le PSTV n'a pu être mis en évidence sur les organes aériens, seule une fraction des tubercules apparaît infectée. La mise en germination des tubercules permet un contrôle supplémentaire au niveau des jeunes feuilles susceptibles d'apporter une confirmation de l'analyse des tubercules.

En bref, l'hybridation moléculaire, qui permet régulièrement de déceler 15 à $30 \mathrm{pg}$ de PSTV purifié, est tout à fait appropriée à une détection précoce du viroïde à la fois au champ mais aussi, ce qui est plus intéressant, dans des opérations de quarantaine ou de contrôle des introductions dans les tubercules ou les jeunes pousses qui en sont issues. Une certaine hétérogénéité de la distribution du PSTV dans la plante conduit, dans l'analyse des tubercules d'une plante, à ne pas limiter l'analyse à un seul tubercule et, si l'on veut une certitude encore plus grande, à confirmer un résultat douteux par une analyse des pousses après germination.

En cas de contrôle de lots importants, les résultats ont montré qu'il était en général difficile d'analyser avec sécurité des lots de plus de 30 tubercules (l'échantillon consistant alors en un germe prélevé sur chacun des tubercules). Là encore, il paraît nécessaire de procéder à un contrôle supplémentaire après germination. Il faut enfin rappeler que l'observation morphologique des tubercules ne peut, en aucun cas, constituer un critère d'infection. Même si, en général, l'infection occasionne une modification au niveau du nombre, de la taille et de la forme, certains tubercules présentent une allure à peu près normale et en outre certaines variétés infectées ont, l'année de l'infection, une production pratiquement normale. C'est dire que des tubercules d'apparence normale peuvent parfaitement être infectés et qu'un indexage très soigneux est nécessaire. La facilité de mise en ouvre de la technique d'hybridation moléculaire nous paraît la placer avant tout autre technique, l'emploi de sondes moléculaires pouvant, dans l'avenir, être facilité par l'utilisation de marquages non radioactifs. 


\section{RÉFÉRENCES BIBLIOGRAPHIQUES}

Bailey J. A., Davidson N., 1976. Methylmercury as a reversible denaturing agent for agarose gel electrophoresis. Anal. Biochem., 70, 75-85.

Kowalska Anne, Skrzeczkowska S., Chrzanowska M., BieleckaPluta D., 1980. Reaction of plants of the potato clone PW 22/70 to potato spindle tuber viroid. Ziemniak. The Potato, 63-77.

Macquaire G., Monsion Marie, Mouches C., Candresse T., Dunez J., 1984. Spot hybridization : application to viroid identification. Ann. Virol., (Inst. Pasteur), 135 (2), 219-230.

Morris T. J., Smith E. M., 1976. Potato spindle tuber disease : procedure for the detection of viroid RNA and certification of diseasefree potato tubers. Phytopathology, 67, 145-150.
Owens R. A., Diener T. O., 1981. Sensitive and rapid diagnosis of potato spindle tuber viroid disease by nucleic acid hybridization. Science, 213, 670-672.

Pfannenstiel M. A., Slack S. A., 1980. Response of potato cultivars to infection by the potato spindle tuber viroid. Phytopathology, 70, 1015-1018.

Schumacher J., Meyer N., Riesner D., Weidemann H. L., 1985. Diagnostic procedure for detection of viroids and viruses with circular RNAs by "return" gel electrophoresis. J. Phytopathol., 115, 332-343. 